\title{
Diagnósticos de enfermagem em urgência e emergência utilizando sistemas de King: estudo descritivo
}

\author{
Nursing diagnoses in urgency and emergency using King's systems: descriptive study \\ Diagnósticos de enfermería en urgencia y emergencia utilizando los sistemas de King: \\ estudio descriptivo
}

Simone Souza Nascimento ${ }^{1 *}$, Cristine Alves Costa de Jesus ${ }^{2}$.

\begin{abstract}
RESUMO
Objetivo: Determinar os diagnósticos de enfermagem da NANDA-I baseados no referencial de King. Métodos: Pesquisa quantitativa, descritiva e transversal, conduzido com 50 participantes. Coleta de dados pela aplicação do Processo de Enfermagem. Os dados obtidos foram analisados por estatística descritiva com auxílio do software Microsoft Excel. O estudo foi aprovado por Comitê de Ética em Pesquisa. Resultados: A maioria dos clientes foi do sexo feminino (54\%), com idade média de 50,48 anos e com baixa escolaridade. Foram identificados 13 fluxogramas, com maior prevalência para Dor torácica (18,0\%). Emergiram 94 diagnósticos de enfermagem, com destaque para Risco de infecção (90,0\%), Conhecimento deficiente $(80,0 \%)$, Risco de quedas $(64,0 \%)$, Estilo de vida sedentário $(62,0 \%)$ e Dentição prejudicada (60,0\%). Conclusão: Os diagnósticos relacionaram-se aos conceitos de pessoa, percepção interação, transação, comunicação, papel, self e imagem corporal, espaço, tempo, estresse, enfermagem e saúde, presentes na teoria de King. Foi possível elaborar reflexões sobre a importância de conhecer a população atendida, bem como a essência do raciocínio clínico e das diferentes habilidades, para a aplicação do processo de enfermagem.
\end{abstract}

Palavras-Chave: Emergências, Diagnóstico de enfermagem, Processo de enfermagem, Teoria de enfermagem, Papel do profissional de enfermagem.

\begin{abstract}
Objective: To determine the NANDA-I nursing diagnoses based on King's framework. Methods: Quantitative, descriptive and transversal research, conducted with 50 participants. Data collection through the application of the Nursing Process. The data obtained were analyzed by descriptive statistics using Microsoft Excel software. The study was approved by the Research Ethics Committee. Results: Most clients were female $(54 \%)$, with a mean age of 50.48 years and with low education. Thirteen flowcharts were identified, with the highest prevalence for Chest pain (18.0\%). Ninety-four nursing diagnoses emerged, with emphasis on Risk of infection (90.0\%), Deficient knowledge (80.0\%), Risk of falls $(64.0 \%)$, Sedentary lifestyle (62.0\%) and Teething impaired (60.0\%). Conclusion: The diagnoses were related to the concepts of person, perception, interaction, transaction, communication, role, self and body image, space, time, stress, nursing and health, present in King's theory. It was possible to elaborate reflections on the importance of knowing the population served, as well as the essence of clinical reasoning and different skills, for the application of the nursing process.
\end{abstract}

Keywords: Emergencies, Nursing diagnosis, Nursing process, Nursing theory, Nurse's role.

\section{RESUMEN}

Objetivo: Determinar los diagnósticos de enfermería de la NANDA-I basados en el marco de referencia de King. Métodos: Investigación cuantitativa, descriptiva y transversal, realizada con 50 participantes. Recolección de datos a través de la aplicación del Proceso de Enfermería. Los datos obtenidos fueron analizados por estadística descriptiva utilizando el software Microsoft Excel. El estudio fue aprobado por el Comité de Ética en Investigación. Resultados: La mayoría de los clientes eran del sexo femenino (54\%),

${ }^{1}$ Escola Superior de Ciências da Saúde, Brasília - DF. *E-mail: enf.simone.sn@gmail.com

2 Universidade de Brasília (UnB), Brasília - DF. 
con edad media de 50,48 años y baja escolaridad. Se identificaron trece diagramas de flujo, con la mayor prevalencia para el dolor torácico $(18,0 \%)$. Emergieron 94 diagnósticos de enfermería, con énfasis en Riesgo de infección $(90,0 \%)$, Conocimiento deficiente $(80,0 \%)$, Riesgo de caídas $(64,0 \%)$, Sedentarismo $(62,0 \%)$ y Dentición perjudicada $(60,0 \%)$. Conclusión: Los diagnósticos se relacionaron con los conceptos de persona, percepción, interacción, transacción, comunicación, rol, imagen del yo y del cuerpo, espacio, tiempo, estrés, enfermería y salud, presentes en la teoría de King. Fue posible elaborar reflexiones sobre la importancia del conocimiento de la población atendida, así como la esencia del raciocinio clínico y diferentes habilidades, para la aplicación del proceso de enfermería.

Palabras clave: Urgencias médicas, Diagnóstico de enfermería, Proceso de enfermería, Teoría de enfermería, Rol de la enfermera.

\section{INTRODUÇÃO}

As emergências referem-se às constatações médicas "de condições de agravo a saúde que impliquem sofrimento intenso ou risco iminente de morte, exigindo tratamento médico imediato, enquanto a urgência é uma ocorrência imprevista de agravo a saúde com ou sem risco potencial a vida, cujo portador necessita de assistência médica imediata" (MINISTÉRIO DA SAÚDE, 2014).

Existe uma crescente demanda por serviços nesta área, devido ao aumento de eventos relacionados aos acidentes, à violência urbana e também às complicações agudas e crônicas das doenças crônicas não transmissíveis. Para tal, Unidades de Pronto Atendimento (UPA) foram planejadas, construídas e implementadas para oferecer atendimento não hospitalar às urgências e emergências. A dinâmica de serviço de uma UPA caracteriza-se por grande rotatividade de usuários, com variados graus de gravidade. Inúmeros fatores podem trazer dificuldades no processo de trabalho nessas unidades, ou mesmo na aplicação do processo de enfermagem, especialmente em decorrência das características do ambiente e da clientela atendida (MINISTÉRIO DA SAÚDE, 2006; VERSA GLGS, et al., 2014; VERAS JEGLF, et al., 2015).

Neste cenário alguns profissionais de enfermagem preocupam-se em sanar apenas os aspectos biológicos do ser humano. Logo, o cuidado fica fragmentado por não considerar o ser humano em sua totalidade e complexidade (BAGGIO MA, et al., 2008).

Por considerar a interação como parte fundamental do cuidado, King elaborou um modelo conceitual abordando sistemas e desenvolveu a Teoria do alcance de metas com base em conceitos a partir do foco central que é o alcance dos objetivos de saúde para o cliente, em uma organização formada por sistemas pessoais, interpessoais e sociais. Neste modelo conceitual supõe-se que a meta da enfermagem tem a finalidade de ajudar o indivíduo a manter sua saúde e consequentemente poder desenvolver atividades e desempenhar seus papeis. King considerava que a interação com os clientes interfere positivamente na finalidade de ajudar o cliente a atingir os objetivos ou metas do cuidado (KING IM, 1981).

Considera-se essencial que o profissional enfermeiro ofereça cuidado científico e planejado, o que exige a adoção do processo de enfermagem fundamentado em um referencial teórico. Tal processo necessita também estar alinhado com os sistemas de classificação dos fenômenos de enfermagem, o que hoje em dia, vislumbra-se no conhecido sistema de classificação dos diagnósticos de enfermagem da North American Nursing Association International (NANDA-I) (HERDMAN TH e KAMITSURU S, 2015).

Assim sendo, conhecer as características da população que frequenta o serviço de saúde é fundamental para o planejamento das ações em saúde, pois, além de favorecer a diminuição das superlotações nos serviços, auxilia os profissionais de saúde no planejamento da assistência, e consequentemente, na melhoria do atendimento, garantindo um cuidado qualificado e com segurança à clientela (OLIVEIRA GN, et al., 2011; RIBEIRO RM, et al., 2014).

Diante do exposto, o objetivo deste estudo foi descrever o perfil sociodemográfico, clínico e epidemiológico dos clientes atendidos em uma Unidade de Pronto Atendimento, admitidos nas Salas Amarela e Vermelha e determinar os diagnósticos de enfermagem da NANDA-I baseados no referencial de King. 


\section{MÉTODOS}

Trata-se de pesquisa de abordagem quantitativa do tipo descritiva, com delineamento transversal. A pesquisa foi realizada nas Salas Amarela e Vermelha de uma Unidade de Pronto Atendimento (UPA) no Distrito Federal.

A população foi composta por indivíduos que buscaram o serviço de pronto atendimento, classificados com prioridade vermelha ou laranja e admitidos nas salas vermelha e amarela, de acordo com o Protocolo Manchester de Classificação de Risco. A amostra foi dimensionada considerando-se um estudo prévio de fluxo de atendimentos com classificação laranja ou vermelha, nos períodos correspondentes do ano anterior, embasado nos dados do prontuário eletrônico e nos livros de registros das respectivas salas. A partir desses dados, mensurou-se inicialmente uma amostra mínima de 40 clientes. Ao final do estudo contou-se com uma amostra de 50 participantes.

A coleta de dados aconteceu de novembro de 2016 a fevereiro de 2017, após o atendimento inicial do indivíduo na unidade de saúde, respeitando o mínimo de três horas de admissão e após esclarecimento da pesquisa e consentimento com assinatura do Termo de Consentimento Livre e Esclarecido (TCLE).

Um instrumento de coleta de dados Foi construído um instrumento de coleta de dados de forma a levantar a caracterização sociodemográfica, de atendimento e clínica do cliente. O instrumento foi composto por perguntas abertas e fechadas, com base no Sistema Conceitual elaborado por King, abordando os três sistemas sabe do modelo: sistema pessoal, interpessoal e social (KING IM, 1981). Dessa forma, foi possível realizar uma abordagem do cliente pautada na visão holística e na classificação da Taxonomia II da NANDA-I (HERDMAN TH e KAMITSURU S, 2015). No instrumento os dados foram agrupados nos sistemas descritos, de forma a levantar os diagnósticos de enfermagem com base nas evidências clínicas, fatores relacionados e fatores de risco elencados.

Três profissionais de enfermagem com experiência mínima de cinco anos no cuidado de pacientes em situação de urgência e emergência e experiência na utilização do Processo de Enfermagem na prática clínica, ensino e na pesquisa, avaliaram o instrumento de coleta de dados, e propuseram alterações para adequação do mesmo. Com os ajustes realizados, realizou-se teste piloto com cinco clientes internados nas salas amarela e vermelha da UPA, que, posteriormente, foram excluídos, não configurando parte da amostra.

A coleta de dados foi realizada a partir da Consulta de Enfermagem, na qual procedeu-se à entrevista com o cliente, seguida da realização de exame físico completo e consulta ao prontuário, mediante o roteiro de coleta de dados desenvolvido. Após a coleta de dados foi realizada a identificação dos Diagnósticos de Enfermagem, utilizando o processo de raciocínio diagnóstico, o qual incluiu as etapas de análise e síntese dos dados coletados do cliente.

Os dados coletados foram digitados em banco de dados e analisados em planilhas eletrônicas no software Microsoft Excel® 2010. As variáveis numéricas foram exploradas pelas medidas descritivas de tendência central (média e mediana) e de dispersão (mínimo e máximo). As variáveis categóricas foram exploradas por frequências simples absolutas e relativas. Os resultados das análises foram organizados em tabelas.

O presente estudo foi aprovado no Comitê de Ética em Pesquisa da Faculdade de Ciências da Saúde da Universidade de Brasília (CEPFS/UnB), sob o Certificado de Apresentação para Apreciação Ética (CAAE) 57475916.8.0000.0030 e pareceres ํㅜ 1.760.638 e oํ 1.861.232, e também pelo Comitê de Ética e Pesquisa da Fundação de Ensino e Pesquisa em Ciências da Saúde da Secretaria de Estado da Saúde do Distrito Federal (CEPFEPECS/SES/DF), sob CAAE 57475916.8.3001.5553 e parecer ํㅜ 1.910.156.

Foi desenvolvido conforme as normas estabelecidas pela resolução oㅜ 466/12 do Conselho Nacional de Saúde, que dispõe sobre pesquisas envolvendo seres humanos, atendendo às exigências éticas e científicas fundamentais, respeitando o participante em seu direito à dignidade, e autonomia, reconhecendo possíveis situações de vulnerabilidade e assegurando sua vontade livre e esclarecidade de contribuir e permanecer ou não na pesquisa. 


\section{RESULTADOS E DISCUSSÃO}

Contou-se com 50 clientes na amostra estudada, com uma margem de confiança de $95 \%$ e margem de erro de 5\% (e). A coleta dos dados ocorreu no período de novembro de 2016 a fevereiro de 2017. Durante 0 período de observação, a taxa de atendimento geral no setor foi de 485 clientes, com 344 altas, 26 óbitos e 115 transferências para outras unidades da rede. Destes, 137 clientes estavam classificados com prioridade muito urgente ou emergente e 50 atenderam os critérios de inclusão no estudo.

O sexo prevalente foi o feminino (27; 54\%). A idade variou entre 18 e 91 anos, com média de 50,48 anos, mediana de 52 anos e moda 61 anos. Houve predominância de indivíduos com idade entre 60 a 69 anos $(15 ; 30 \%)$. Em relação à escolaridade, a amostra caracteriza-se por baixa escolaridade, uma vez que $23(46 \%)$ destes não concluíram o ensino fundamental e sete (14\%) não eram alfabetizados. Quanto à raça/cor autodeclarada, predominou-se a parda (22; 44\%). A prevalência de renda mensal era de 1 a 2 salários mínimos (32; 64\%). No entanto, 36\% estavam desempregados. A maioria também declarou estar com companheiro (36; 72\%).

A Tabela 1 apresenta a caracterização do total de clientes que receberam as classificações urgente e emergente durante o período de observação.

Tabela 1 - Caracterização do perfil socioeconômico-demográfico da amostra de clientes com classificações muito urgente e emergente atendidos nas salas de observação, $n=50$. Brasília - DF, novembro 2016 a fevereiro 2017.

\begin{tabular}{|c|c|c|}
\hline Característica & $\mathbf{N}$ & $\%$ \\
\hline \multicolumn{3}{|l|}{ Sexo } \\
\hline Feminino & 27 & 54,0 \\
\hline Masculino & 23 & 46,0 \\
\hline \multicolumn{3}{|l|}{ Escolaridade } \\
\hline Não alfabetizado & 7 & 14,0 \\
\hline Fundamental Completo & 9 & 18,0 \\
\hline Fundamental Incompleto & 23 & 46,0 \\
\hline Médio Completo & 6 & 12,0 \\
\hline Médio Incompleto & 3 & 6.0 \\
\hline Superior Incompleto & 2 & 4,0 \\
\hline \multicolumn{3}{|l|}{ Faixa etária } \\
\hline$<20$ & 1 & 2,0 \\
\hline $20-29$ & 6 & 12,0 \\
\hline $30-39$ & 10 & 20,0 \\
\hline $40-49$ & 6 & 120 \\
\hline $50-59$ & 9 & 18,0 \\
\hline $60-69$ & 15 & 30,0 \\
\hline 70 ou mais & 3 & 6,0 \\
\hline \multicolumn{3}{|l|}{ Raça/cor } \\
\hline Branca & 17 & 34,0 \\
\hline Parda & 22 & 44,0 \\
\hline Preta & 10 & 20,0 \\
\hline Indígena & 1 & 2,0 \\
\hline \multicolumn{3}{|l|}{ Renda familiar } \\
\hline$<1$ salário & 6 & 12,0 \\
\hline 1 a 2 salários & 32 & 64,0 \\
\hline 3 a 5 salários & 12 & 24,0 \\
\hline \multicolumn{3}{|l|}{ Ocupacão/Fonte de Renda } \\
\hline Assalariado & 22 & 44,0 \\
\hline Aposentadoria & 10 & 20,0 \\
\hline Desempregado & 18 & 36,0 \\
\hline \multicolumn{3}{|l|}{ Estado conjugal } \\
\hline Solteiro & 6 & 12,0 \\
\hline Casado/amasiado & 36 & 72,0 \\
\hline Divorciado/separado & 5 & 10,0 \\
\hline Viúvo & 3 & 6,0 \\
\hline
\end{tabular}

Fonte: Nascimento SS e Jesus CAC, 2022. 
Foram arrolados ao todo 722 Diagnósticos de Enfermagem (DE) nos 50 indivíduos estudados, considerando que os diagnósticos se repetiam a cada indivíduo, com variação de 5 a 25, média de 14,44 e mediana de 15 diagnósticos por cliente. Obteve-se 94 rótulos diagnósticos diferentes, sendo 54 diagnósticos com foco no problema (reais), dois diagnósticos de promoção da saúde e 38 de risco (ou vulnerabilidade), distribuídos entre 12 domínios da NANDA-I. Dos DE estudados, cinco tiveram frequências iguais ou superiores à 50\%, conforme Tabela 2.

Tabela 2 - Diagnósticos de enfermagem com foco no problema, promoção da saúde e de risco nos clientes com classificações muito urgente e emergente atendidos nas salas de observação, $n=50$. Brasília - DF, novembro 2016 a fevereiro 2017.

\begin{tabular}{lcc}
\hline Diagnóstico de Enfermagem & $\mathbf{N}$ & $\%$ \\
\hline Risco de Infecção & 45 & 90,0 \\
Conhecimento deficiente & 40 & 80,0 \\
Risco de quedas & 32 & 64,0 \\
Estilo de vida sedentário & 31 & 62,0 \\
Dentição prejudicada & 30 & 60,0 \\
\hline
\end{tabular}

Fonte: Nascimento SS e Jesus CAC, 2022.

Risco de infecção foi o DE com maior prevalência (90\%). É definido como "vulnerabilidade à invasão e multiplicação de organismos patogênicos, que pode comprometer a saúde" (HERDMAN TH e KAMITSURU S, 2015).

Este diagnóstico foi evidenciado em $90 \%$ da amostra deste estudo, e os fatores de risco associados foram: procedimento invasivo $(97,8 \%)$, enfermidade crônica $(40,0 \%)$, obesidade $(33,3 \%)$, tabagismo $(11,1 \%)$, desnutrição e diminuição da hemoglobina $(6,7 \%$, cada), alteração na integridade da pele e alteração no peristaltismo (4,4\%, cada) e imunossupressão $(2,2 \%)$.

Ao se pensar em procedimentos invasivos como fator de risco para infecção, cabe ressaltar que a maioria destes foi realizado pelo profissional de enfermagem, sendo punções venosas periféricas (94\%) e cateterismos vesicais (14\%). Procedimentos realizados por outros profissionais também foram considerados, como punção de acesso venoso central (4\%) ou toracocentese $(2 \%)$.

Assim sendo cabe discutir a importância do papel do profissional de enfermagem no momento de execução da técnica, assegurando as medidas de assepsia durante todo o procedimento, incluindo o preparo do material bem como a higienização das mãos, bem como as definições de manutenção e periodicidade de troca de dispositivos, como forma de prevenir infecções (DANSKI MT, et al., 2016).

Nos serviços de urgência e emergência os riscos inerentes à prestação da assistência de enfermagem aumentam consideravelmente o índice de infecções, visto que os profissionais e clientes estão expostos em um ambiente que favorece o surgimento de infecções, bem como pode ser consequência da grande demanda de pacientes, do despreparo dos profissionais, da falta de recursos humanos e materiais e da não adesão às práticas propostas (VALLE ARMC, et al, 2008). Considerando os aspectos abordados é possível relacionar este diagnóstico aos conceitos de pessoa, espaço, tempo, enfermagem, estresse e saúde da teoria de King IM (1981).

O segundo DE prevalente na amostra foi Conhecimento deficiente, definido como "ausência ou deficiência de informação cognitiva relacionada a um tópico específico" (HERDMAN TH e KAMITSURU S, 2015).

Durante a coleta de dados, foi questionado ao cliente sobre patologias pregressas, medicações em uso contínuo, motivo da internação e tratamento na internação. As respostas negativas ou incompletas a estas questões permitiram levantar este diagnóstico. Além disso, os clientes demonstravam falta de acompanhamento de doenças crônicas, que reforça a inferência de escassos conhecimentos relativos ao tratamento e à doença. Outros aspectos não conhecidos por parte da clientela, como as complicações e forma de controle da DCNT, bem como uso correto das medicações além de medidas não medicamentosas, e também sobre a saúde dental, permitiram levantar inferências sobre o déficit do conhecimento nas mesmas. 
Cabe ressaltar que a baixa escolaridade foi uma evidência da clientela deste estudo, e este fator também pode estar relacionado à capacidade de assimilação de novas informações, especialmente no que diz respeito ao reconhecimento dos medicamentos em uso, principalmente nos casos de polimedicação, evento também evidenciado neste estudo.

Além disso, a comunicação, o diálogo e a conversa, estabelecida entre o profissional da saúde e o cliente é essencial para a aproximação entre ser cuidado e o ser cuidador. A partir da relação e da interação humana, propostos por King IM (1981), momentos de troca, de interesse e preocupação podem refletir em uma parceria indissolúvel no processo do cuidado (BAGGIO MA, et al., 2008). Ainda acerca da teoria de King IM (1981), é possível observar a presença dos conceitos de pessoa, percepção interação, transação, comunicação, enfermagem e saúde neste diagnóstico.

Estilo de vida sedentário foi o terceiro DE mais prevalente e "refere-se a um hábito de vida que se caracteriza por um baixo nível de atividade física" (HERDMAN TH e KAMITSURU S, 2015). Um estudo aponta que o sedentarismo em adolescentes está associado com residir em área urbana. Com a urbanização como realidade é necessário investir em ações de saúde para esta população, uma vez que serão os adultos e idosos do futuro (REGIS MF, 2016). Relacionando com a teoria e King IM (1981), são percebidos os conceitos de pessoa, papel, percepção, interação, comunicação, transação e saúde neste diagnóstico.

Dentição prejudicada foi um DE prevalente em $60 \%$ da amostra, e é definido como "ruptura nos padrões de desenvolvimento/erupção dentário ou na integridade estrutural de cada dente" (HERDMAN TH e KAMITSURU S, 2015).

$\mathrm{Na}$ clientela estudada foram elencadas as seguintes características definidoras: ausência de dentes, dentes desgastados, descoloração do esmalte, halitose e dentes frouxos. Ainda foram identificados os fatores relacionados: dificuldade de acesso a cuidados dentários profissionais, conhecimento insuficiente sobre a saúde dental, economicamente desfavorecido, hábitos alimentares inadequados, desnutrição e higiene oral inadequada.

O conhecimento insuficiente acerca da correta higiene oral e os cuidados precários com a higiene são aspectos presentes em outros estudos. Além disso, há relação do déficit no conhecimento a falta de conhecimento dos pais na fase da infância dos filhos e até mesmo da pessoa que presta cuidado ao cliente, além da carência de introdução de práticas educativas nas atividades escolares, bem como da implantação de programas de prevenção adequados à realidade e ao nível socioeconômico, e cultural das comunidades, o que pode ser um fator agravante nas etapas subsequentes do desenvolvimento humano (BALDANI MH, et al., 2010; AFONSO BA e CASTRO MCC, 2014; SANTOS MA, et al., 2015).

A situação financeira também relatada como dificuldade para acesso, pode ser confirmada ao se levar em consideração que a maior parte da amostra deste estudo tinha renda familiar de até dois salários mínimos. Além disso, deve-se considerar o déficit do autocuidado, que abrange as condições de higiene bucal, que por sua vez estão relacionadas aos cuidados de enfermagem. Mesmo que o indivíduo tenha autonomia, é tarefa da enfermagem realizar avaliação e orientação diariamente, enquanto o cliente está sob seus cuidados.

No entanto, apenas a criação de protocolos não garante melhora nos cuidados bucais prestados pelos profissionais. É necessário que também ocorra educação continuada eficiente e avaliações da qualidade de assistência prestada, pois, uma vez que existem falhas nesse âmbito do cuidado, isto pode contribuir para complicações infecciosas na evolução clínica do paciente, comprometendo a segurança do mesmo e a qualidade da assistência prestada (NOGUEIRA JWS, 2016).

Em relação à teoria proposta neste estudo, cabe ressaltar mais uma vez a importância da interação, bem como a aplicabilidade de outros conceitos da teoria como a pessoa, percepção, comunicação, interação, self e imagem corporal (KING IM, 1981).

Por fim, mas não menos importante, Risco de quedas é definido como "Vulnerabilidade ao aumento da susceptibilidade a quedas, que pode causar dano físico e comprometer a saúde" (HERDMAN TH e KAMITSURU S, 2015). 
Este diagnóstico foi identificado em $64 \%$ dos clientes estudados. Os fatores de risco de maior incidência no estudo foram: deficiência visual $(78,1 \%)$, dificuldades na marcha $(31,3 \%)$, ausência de sono $(28,1 \%)$, agente farmacológico $(28,1 \%)$, idade maior ou igual a 65 anos $(25,0 \%)$, e mobilidade prejudicada $(21,9 \%)$.

Neste estudo, utilizou-se a Escala de Risco de Quedas de Morse JM, et al. (1989). Todos os indivíduos apresentaram algum risco para quedas, mas $28 \%$ destes apresentaram alto risco (pontuação maior que 50 ). A pontuação média foi de 38,8 pontos. Dentre os itens avaliados na escala seis clientes tinham histórico de quedas nos últimos três meses, item que confere 25 pontos; 42 clientes possuíam algum diagnóstico secundário, o que confere 15 pontos, quatro necessitavam de auxílio na deambulação, dos quais dois necessitavam de pouca assistência e dois apresentavam moderada dependência, o que confere 15 e 30 pontos respectivamente; 47 estavam em terapia endovenosa contínua ou com dispositivo salinizado, conferindo 20 pontos; 11 clientes tinham alteração na marcha, dos quais nove era pequena (10 pontos) e dois moderada a grave (20 pontos). Quanto à avaliação do estado mental, todos os clientes somaram zero ponto, uma vez que não houve alteração.

A média de idade dos clientes que apresentaram risco de quedas foi de 58,5 anos. Metade destes indivíduos tinham 60 anos ou mais de idade, o que chama a atenção para a alta prevalência adultos e adultos jovens com risco de quedas neste estudo.

Quedas de pacientes no ambiente hospitalar são um grande problema para as instituições de saúde, pois podem repercutir no tempo de internação, piora do quadro clínico e até mesmo a morte, além das consequências emocionais e sociais. Dessa forma, é importante avaliar os fatores de risco, a fim de estabelecer estratégias de prevenção de quedas que orientem a equipe de profissionais, bem como clientes e familiares, a partir da admissão dos pacientes, uma vez que constitui um fator fundamental para a promoção da segurança do paciente no desenvolvimento do processo de enfermagem (VITOR AF e ARAÚJO TL, 2011). A partir deste contexto é possível relacionar este diagnóstico aos conceitos de pessoa, espaço, enfermagem, interação, estresse e saúde da teoria de King IM (1989).

\section{CONCLUSÃO}

A articulação sistêmica entre cliente, enfermeiro, organização social e processo de enfermagem é um grande desafio, considerando a assistência de enfermagem em geral, até mesmo para a Enfermagem moderna. Quando se trata da assistência ao paciente em unidades de emergência com o processo de enfermagem adequado, é ainda mais complexo e desafiador que tal articulação ocorra, principalmente considerando as questões relacionadas à ambiência, alto fluxo e demanda e força de trabalho menor que a necessária para assistência de qualidade. Dessa forma, é necessária que a atuação do enfermeiro seja fundamentada em conhecimento científico e evidenciada por um processo de enfermagem claro, completo e preciso acerca da assistência a ser prestada. A realização deste estudo permitiu elencar reflexões da importância do desenvolvimento do raciocínio clínico, considerando as diferentes habilidades necessárias para tal. A aplicação do processo de enfermagem exige, entre muitas coisas, o olhar e o cuidar holísticos. Espera-se que este estudo contribua com a construção do conhecimento da enfermagem e, em especial, com o processo da implementação da Sistematização da Assistência de Enfermagem, demonstrando cada vez mais a cientificidade desta profissão e área do saber. Espera-se também que contribua com 0 cuidado ao paciente que utiliza este e demais serviços de saúde similares.

\section{REFERÊNCIAS}

1. AFONSO BA, CASTRO MCC. Evaluation of oral hygiene knowledge and parent motivation in a Brazilian public education institution. Arq. Odontol., 2014; 50(4): 161-169.

2. BAGGIO MA, et al. Understanding care dimensions in an emergency hospital unit. Rev. Bras. Enferm., 2008; 62(3): 552-557.

3. BALDANI MH, et al. Individual determinants of dental care utilization among low-income adult and elderly individuals. Rev. Bras. Epidemiol., 2010; 13(1): 150-162.

4. DANSKI MT, et al. Complications related to the use of peripheral venous catheters: a randomized clinical trial. Acta paul. Enferm., 2016; 29(1): 84-92. 
5. HERDMAN TH, KAMITSURU S. Diagnósticos de enfermagem da NANDA-I: definições e classificação 2018-2020. $11^{\underline{a}}$ ed. Porto Alegre: Artmed, 2015.

6. KING IM. A Theory for Nursing: systems, concepts, process. USA: Delmar Publishers Inc, 1981.

7. MINISTÉRIO DA SAÚDE. Política nacional de atenção às urgências. 2006. Disponível em: http://bvsms.saude.gov.br/bvs/publicacoes/politica_nacional_atencao_urgencias_3ed.pdf Acessado em: 26 de agosto de 2020.

8. MINISTÉRIO DA SAÚDE. Portaria № 354, de 10 de março de 2014. Publica a proposta de Projeto de Resolução Boas Práticas para Organização e Funcionamento de Serviços de Urgência e Emergência. 2014 Disponível em: http://bvsms.saude.gov.br/bvs/saudelegis/gm/2014/prt0354_10_03_2014.html Acessado em: 26 de agosto de 2020.

9. MORSE JM, et al. Development of a scale to identify the fall-prone patient. Canadian Journal on Aging, 1989; 8(4): 366-377.

10. NOGUEIRA JWS. Nursing team practice in the preventive oral hygiene of caredependent patients. (Mestrado em Enfermagem) - Faculdade de Ciências da Saúde, Universidade de Brasília, Brasília, 2016; 148p.

11. OLIVEIRA GN, et al. Profile of the Population Cared for in a Referral Emergency Unit. Rev. Latino-Am. Enfermagem, $2011 ; 19(3)$.

12. REGIS MF, et al. Urban versus rural lifestyle in adolescents: associations between environment, physical activity levels and sedentary behavior. Einstein, 2016; 14(4): 461-467.

13. RIBEIRO RM, et al. Profile of clinical emergencies in the emergency department of a teaching hospital. REME Rev. Min. Enferm., 2014; 18(3): 533-538

14. SANTOS MA, et al. Oral health knowledge and perception of teachers and preschoolers from a city in the state of Bahia, Brazil. RFO Revista Da Faculdade De Odontologia, 2015; 20(2): 172-178.

15. VALLE ARMC, et al. Social Representations of Biosecurity by Nursing Professionals at an Emergency Service. Esc. Anna Nery Rev. Enferm., 2008; 12(2): 304-309.

16. VERAS JEGLF, et al. Evaluation of nursing skills to promote health during pediatric consultations in emergency rooms. Acta Paul. Enferm., 2015; 28(5): 467-474.

17. VERSA GLGS, et al. Assessment of user embracement with risk rating in emergency hospital services. Rev. Gaúcha Enferm., 2014; 35(3): 21-28.

18. VITOR AF, ARAÚJO TL. Definitions for the nursing outcome behavior for falls prevention: an integrative review. Rev. Eletr. Enf., 2011; 13(2): 313-322. 